\title{
Biofeedback therapy for constipation due to dyssynergic defecation: ready for prime time
}

\author{
G. Chiarioni $^{1,3} \cdot$ G. Bassotti ${ }^{2}$
}

Received: 28 March 2015 / Accepted: 28 March 2015/Published online: 12 May 2015

(C) Springer-Verlag Italia Srl 2015

Chronic constipation is a common, benign condition with considerable impact on health expenses and quality of life [1]. The majority of patients usually respond to diet and lifestyle modifications or to judicious use of laxatives; among the non-responders, outlet dysfunction-type constipation seems particularly common, affecting up to $50 \%$ of patients referred to tertiary care centers [1]. Most of these patients will show feature of dyssynergic defecation (i.e., paradoxical contraction or failure to relax the pelvic floor and anal muscles during defecation) leading to inadequate emptying of the rectum [2]. Dyssynergic defecation is thought to be a maladaptive learned behavior, since usually no identifiable neurological or anatomical defect is found, and the condition has been found to resolve after patients undergo behavioral training [3]. Biofeedback therapy to teach effective defecation effort has been extensively used in obstructed defecation syndrome [1]. However, the scientific evidence favoring biofeedback over conservative treatment for dyssynergic defecation has been controversial up to the past decade [1]. Major obstacles when assessing meaningful literature were the huge variety of inclusion and outcome criteria, follow-up intervals, and therapeutic protocols [3]. Recently, three randomized control trials (RCTs) have proven that biofeedback is an effective

G. Bassotti

gabassot@tin.it

1 Gastroenterology Division, University of Verona, AOUI Verona, Verona, Italy

2 Gastroenterology and Hepatology Section, Department of Medicine, University of Perugia Medical School, Piazza Lucio Severi, 1, 06132 San Sisto, Perugia, Italy

3 Center for Functional GI and Motility Disorders, University of North Carolina at Chapel Hill, Chapel Hill, NC, USA treatment for dyssynergic defecation when compared to placebo, laxatives (including macrogol), and muscle relaxant drugs [2, 4]. However, generalization of results was hampered by patient selection, rigid outcome criteria, and the administration of therapy by highly specialized referral centers, focused on dyssynergic defecation [2, 3]. Moreover, it was unclear whether the positive outcome reported by some authors was related to the expertise of the care providers [2,3]. Some of these limitations have been effectively addressed by the RCT study appearing in this issue that compared augmented biofeedback therapy for dyssynergic defecation to scheduled counselling sessions [5]. Randomization was well designed, without excluding patients with slow transit constipation. Another point worth mentioning is the inclusion of patients who had previously undergone ineffective surgery for obstructed defecation, as in "real life". This also prompts a word of caution about the surgical options in this disabled group of patients. The number of treatment sessions chosen (six) is likely to be cost-effective at any center. The choice of outcome criteria is optimal for the surgical and the gastroenterological audience, for the Wexner score is one of the most commonly used scoring systems to assess outcomes and sound data about the efficacy of biofeedback therapy on improving quality of life (QOL) scores are lacking. Finally, behavioral treatment has been provided in a surgical center, likely more focused on invasive treatment options. Nonetheless, augmented biofeedback has been clearly shown to be more effective than control treatment in improving symptoms and QOL scores in constipation secondary to dyssynergic defecation. Unfortunately, anorectal testing follow-up did not include evaluation of defecation behavior to confirm whether the effectiveness of biofeedback therapy is related to restored physiology. It is unclear, as the authors honestly acknowledge, whether augmented biofeedback therapy is 
more effective than simple biofeedback therapy. However, adding a standardized myorelaxant treatment of the pelvic floor to behavior therapy is likely to be helpful outside of biofeedback referral centers.

In conclusion, biofeedback treatment has been proven to be an effective therapy for severe, unresponsive constipation secondary to dyssynergic defecation. This approach should be considered a viable option in any patient referred for this behavioral disorder, as it pertains to both previous pelvic floor surgery and associated colon transit disorder. Improvement in symptoms mirrors amelioration in QOL, providing additional motivation when considering this treatment option with the affected patient. The biofeedback treatment approach should not be limited to referral centers.

Conflict of interest None.

\section{References}

1. Bassotti G, Chistolini F, Sietchiping-Nzepa F, De Roberto G, Morelli A, Chiarioni G (2004) Biofeedback for pelvic floor dysfunction in constipation. BMJ 382:393-396

2. Chiarioni G, Heymen S, Whitehead WE (2006) Biofeedback therapy for dyssynergic defecation. World J Gastroenterol 12:7069-7074

3. Chiarioni G, Whitehead WE (2008) The role of biofeedback in the treatment of gastrointestinal disorders. Nat Clin Pract Gastroenterol Hepatol 5:371-382

4. Chiarioni G, Whitehead WE, Pezza V, Morelli A, Bassotti G (2006) Biofeedback is superior to laxatives for normal transit constipation due to pelvic floor dyssynergia. Gastroenterology 130:657-664

5. Cadeddu F, Salis F, De Luca E, Ciangola I, Milito G (2015) Efficacy of biofeedback plus transanal stimulation in the management of pelvic floor dyssynergia: a randomized trial. Tech Coloproctol. doi:10.1007/s10151-015-1292-7 